\title{
Moving magic as hospitals merge in Montréal
}

W

hen one emergency department closes, another opens. That's the plan in Montréal, Quebec, anyway, when the McGill University Health Centre (MUHC) moves to a new superhospital in 2015.

Moving one hospital is a colossal feat of logistics. The MUHC, however, is planning multiple large-scale hospital relocations and is shuffling selected departments and facilities from one site to another.

The new site will house the Montreal Children's Hospital, the MUHC Research Institute, parts of the Montreal General Hospital, the Royal Victoria Hospital, the Shriners Hospital for Children Canada and the Montreal Chest Institute, and a new cancer centre.

Meanwhile, parts of the Royal Victoria Hospital will relocate to the Montreal General Hospital, which will remain open as a trauma centre, and parts of the Chest Institute will relocate to the MUHC's community hospital in Lachine, Que.

The new $223000 \mathrm{~m}^{2}$ superhospital, which will be the biggest in Canada, includes 500 single-patient rooms and two emergency departments. Located at the intersection of the Notre-Damede-Grâce, Sud Ouest and Westmount neighbourhoods in Montréal, the mammoth facility will cost an estimated $\$ 1.3$ billion to build. For several years it has been the largest construction project in North America.

Several of the hospital buildings it's replacing are more than 100 years old and can't be updated to meet current standards. The new superhospital at the Glen site is a solution to this problem, and planners expect it will make the MUHC more efficient by combining services that are replicated in individual hospitals, such as the laboratory, pharmacy, housekeeping and kitchens. Emergency departments, operating rooms and imaging suites will also be collocated.

Moving hospitals, departments and patients will have to be "very, very well coordinated," says Imma Franco, the

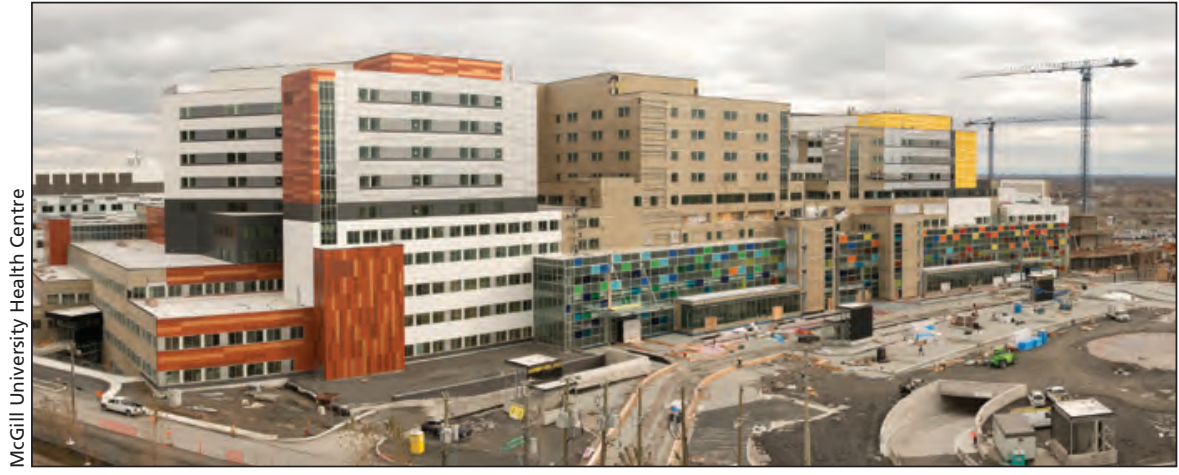

The new $223000 \mathrm{~m}^{2}$ superhospital in Montréal will be the largest in Canada. It includes 500 single-patient rooms and two emergency departments.

MUHC's associate director for planning of programs and services, so as not to interrupt essential health care services.

\section{Moving plan}

The current plan is for the Research Institute to move first in February 2015, followed by the Royal Victoria in April. The latter is the biggest hospital, "so it should get the building up and running well," says Michèle Lortie, the Glen Activation project director. Each hospital has two weeks to move, and then two weeks to settle in before the next hospital arrives.

The Royal Victoria will be followed by the Montreal Children's Hospital, the Chest Institute and components of the Montreal General. If all goes according to plan, this phase of the move should be finished in mid-June, 2015.

On the final Sunday of each facility's move, patients will make the journey to the new site accompanied by a medical team. Ambulances and shuttle busses will help patients move. Certain roads will likely be closed to ensure travel goes smoothly.

The hospitals plan to have the fewest possible patients at the time of the move. "We will have stopped elective surgeries several days before, so our census should be as low as it could be, but inevitably there will be patients in our intensive care units, in our neonatal intensive care units, that cannot go home or to another facility," says Franco.

Franco and Lortie provide oversight for the enormous project, but Health Care Relocations, a company based in Peterborough, Ontario, with two decades of experience in hospital relocations, will be facilitating the move.

"We have to plan for the worst and then it'll be easy," says Franco. "You plan for all the possible scenarios patient codes, what you do, all those things. The planning takes a lot of time and a lot of effort, and once you develop the plan the key thing is people need to stick to the plan."

There should be no shortage of helping hands. When the Royal Victoria Hospital moves, the Children's Hospital will send people to help, and so on.

"It's not very often that anybody who works in health care goes through an experience like this. They'll all rise to the occasion," Franco says.

In the meantime, the MUHC is preparing its staff to work in a new setting with new technology and new teams after the move. "There's a major transformation that occurs in preparation for the move. So, not only are the teams being asked to learn a new environment, but they're being asked to change the way they practise," Franco says.

New MUHC-wide protocols for things such as infection prevention and control are also being rolled out in preparation for the move to the new facility. Catherine Cross, Ottawa, Ont.

CMAJ 2014. DOI:10.1503/cmaj.109-4709 